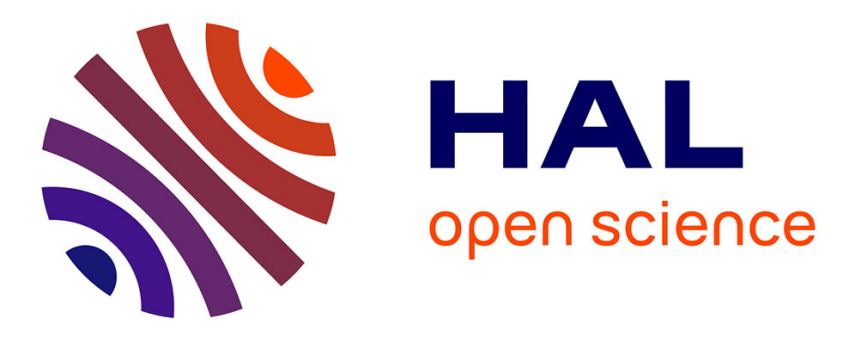

\title{
Improved stability criteria for sampled-data systems with input saturations
}

Alexandre Seuret, Frédéric Gouaisbaut, Sophie Tarbouriech, Joâo Manoel

Gomes da Silva Gomes da Silva

\section{To cite this version:}

Alexandre Seuret, Frédéric Gouaisbaut, Sophie Tarbouriech, Joâo Manoel Gomes da Silva Gomes da Silva. Improved stability criteria for sampled-data systems with input saturations. Recent Results on Nonlinear Delay Control Systems, pp.311-325, 2015, 978-3-319-18071-7. 10.1007/978-3-319-180724_16. hal-00992750

\section{HAL Id: hal-00992750 \\ https://hal.science/hal-00992750}

Submitted on 19 May 2014

HAL is a multi-disciplinary open access archive for the deposit and dissemination of scientific research documents, whether they are published or not. The documents may come from teaching and research institutions in France or abroad, or from public or private research centers.
L'archive ouverte pluridisciplinaire HAL, est destinée au dépôt et à la diffusion de documents scientifiques de niveau recherche, publiés ou non, émanant des établissements d'enseignement et de recherche français ou étrangers, des laboratoires publics ou privés. 


\title{
Chapter 1 \\ Improved stability criteria for sampled-data systems with input saturations
}

\author{
Alexandre Seuret, Frédéric. Gouaisbaut, Sophie Tarbouriech and Joao M. Gomes \\ da Silva Jr.
}

\begin{abstract}
In this chapter, the design of either the controller or the network is addressed for sampled-data systems with input saturation. Using modified sector conditions, an adequate looped functional, and the Wirtinger-based integral inequality, quasi-LMI conditions, with a scalar parameter to tune, are proposed in the regional (or local) context for both design problems. The associated convex optimizations are briefly described. Finally some examples show the efficiency of the methods with respect to existing results.
\end{abstract}

\subsection{Introduction}

In the past decade, a large attention has been devoted to the stability analysis of sampled-data systems [10]. This area addresses the problem of stability and stabilization of systems evolving in continuous time whereas the control input evolves in discrete time. Although the theory for linear sampled-data control systems is well established for constant sampling period [2] through the discretization of the continuous-time systems, there are still a lot of activities regarding the case of asynchronous sampling. Indeed in practice, a heavy temporary load in the hardware

A. Seuret and S. Tarbouriech

CNRS, LAAS, 7 avenue du Colonel Roche 31400 Toulouse, France and Univ de Toulouse, LAAS

F-31400 Toulouse France, e-mail: seuret,tarbour@laas.fr

F. Gouaisbaut

UPS, LAAS, 7 avenue du Colonel Roche 31400 Toulouse, France and Univ de Toulouse, LAAS

F-31400 Toulouse France, e-mail: gouaisb@laas.fr

J.M. Gomes Da Silva Jr.

UFRGS Departamento de Engenharia Elétrica, Av. Osvaldo Aranha 103, esq. Sarmento Leite 90035-051 Porto Alegre-RS, Brasil e-mail: jmgomes@ece.ufrgs.br

This work was supported by ANR project LimICoS contract number 12 BS03 00501 and by STIC-AmSud project ADNEC." 
which compute the control inputs can lead to significant variations on the sampling time. These variations can dramatically affect the stability properties of a control system. That is then clearly the motivation for the design of robust stability and stabilization conditions which take into account the variations on the sampling period.

Among the possible solution, the sampling in a control loop can be modeled as a time-varying delay on the plant control input while considering a continuous-time dynamics. In this case, the delay variation rate between two sampling instants is equal to one. Thus, a fundamental problem regards the determination of the bounds on the delay variation, for which stability of the closed-loop system can be kept. Considering this perspective, we can cite for example the approach proposed in [5], and the improvements made in [17] and [4].

On the other side, many techniques have been proposed in the literature to deal with the stabilization of time-delay systems subject to actuator saturation. In this context, we can cite, for instance: [18] and [19], where globally stabilizing control laws are proposed; and [27], [3] and [5], where the regional stabilization problem is considered; $[15,16]$, where the authors consider the problem of globally stabilizing systems with bounded feedbacks in the case where is present an arbitrarily large delay in the input. These works are mainly concerned by state delayed systems. The stabilization conditions consider delay independent approaches and delay dependent conditions considering fixed delays. Recently, in [7] and [30], results considering systems presenting time-varying delays on the states have been proposed. On the other hand, we can note a lack of results considering input delays, and, in particular, time-varying ones. In [9] and [28], anti-windup techniques are proposed for systems with fixed input delays.

In this chapter we are interested in the problem of stabilizing a sampled-data system taking into account the possible variations on the sample intervals which are possibly due to packet losses in a communication network and also the fact that the signals provided by the actuators are bounded (i.e saturating plant inputs). The method is based on the use of a particular functional that, differently from the Lyapunov-Krasovskii based approaches adopted for instance in [5], [17] and [4], does not need to be positive definite. It is shown that if the time-derivative of this functional along the trajectories of the continuous-time model is strictly negative, then a quadratic Lyapunov function is strictly decreasing for the discrete-time asynchronous system. Such an analysis has already been addressed in [22]. In the present chapter, we aim at presenting an improved version of the stability conditions in [22] by using of a less conservative integral inequality than the usually Jensen inequality. This new integral inequality which is inspired from [23], is adapted to our purpose in order to fit with the proposed analysis. On the other hand, the control saturation is taken into account from the use of a generalized sector condition.

The paper is organized as follows. Section 1.2 describes the problem formulation. Section 1.3 presents some preliminary lemmas on a LMI manipulation and on the generalized sector condition. Section 1.4 shows the main stabilization theorem. In Section 1.5, some optimization problems are proposed. Some numerical examples illustrating the potentialities of the method are provided in Section 1.6. 
Notation. Throughout the chapter, the sets $\mathbb{N}, \mathbb{R}^{+}, \mathbb{R}^{n}, \mathbb{R}^{n \times n}$ and $\mathbb{S}^{n}$ denote respectively the set of positive integers, positive scalars, $n$-dimensional vectors, $n \times n$ matrices and symmetric matrices of $\mathbb{R}^{n \times n}$. For a given positive scalar, $T_{\max }$, define $\mathbb{K}$ as the set of continuous functions from an interval $[0, T]$ to $\mathbb{R}^{n}$, where $T$ is a positive scalar less than $T_{\max }$. The notation $|\cdot|$ and $\|\cdot\|$ stand for the absolute value of a scalar and for the Euclidean norm of a vector, respectively. The superscript ' $T$ ' stands for matrix or vector transposition. The notation $P>0$ for $P \in \mathbb{S}^{n}$ means that $P$ is symmetric positive definite. For any positive integer $j \leq n$ any vector $x \in \mathbb{R}^{n}$ and any matrix $A \in \mathbb{R}^{n \times n}$, the notation $A_{j}$ and $x_{j}$ refer to the $j^{\text {th }}$ line of matrix $A$ and the $j^{\text {th }}$ component of vector $x$, respectively. The symbols $I$ and 0 represent the identity and the zero matrices of appropriate dimensions. $\operatorname{Co}\{\cdot\}$ denotes a convex hull. For any matrix $A$ in $\mathbb{R}^{n \times n}$, we define $\operatorname{He}\{A\}$ as $A+A^{T}$.

\subsection{Problem formulation}

Let $\left\{t_{k}\right\}_{k \in \mathbb{N}}$ be an increasing sequence of positive scalars such that $\bigcup_{k \in \mathbb{N}}\left[t_{k}, t_{k+1}[=\right.$ $\left[0,+\infty\left[\right.\right.$. Assume that there exist two positive scalars $T_{\min } \leq T_{\max }$ such that the difference between two successive sampling instants $T_{k}=t_{k+1}-t_{k}$ satisfies

$$
\forall k \in \mathbb{N}, \quad 0 \leq T_{\min } \leq T_{k} \leq T_{\max } .
$$

Consider the linear system with a sampled-data input

$$
\forall t \in\left[t_{k}, t_{k+1}\left[, \quad \dot{x}(t)=A x(t)+B u\left(t_{k}\right),\right.\right.
$$

where $x \in \mathbb{R}^{n}$ and $u \in \mathbb{R}^{m}$ represent the state variable and the input vector. The matrices $A$ and $B$ are assumed to be constant, known and of appropriate dimensions. We suppose that the input vector $u$ is subject to amplitude limitations defined by:

$$
\left|u_{i}\right| \leq u_{0 i}, \quad u_{0 i}>0, i=1, \ldots, m .
$$

Consider a linear state feedback control law $u(t)=K x(t)$, where $K \in \mathbb{R}^{m \times n}$. Due to the control bounds defined in (1.3), the effective control signal to be applied to the system is given by

$$
u(t)=\operatorname{sat}(K x(t)),
$$

with $\left.u_{i}(t)=\operatorname{sat}\left(K_{i} x(t)\right)=\operatorname{sign}\left(K_{i} x(t)\right)\right) \min \left\{u_{0 i},\left|K_{i} x(t)\right|\right\}, i=1, \ldots, m$. Hence, the closed-loop system reads

$$
\dot{x}(t)=A x(t)+B s a t\left(K x\left(t_{k}\right)\right) .
$$

Although the system dynamics are considered to be linear, due to the control saturation, the closed-loop system is nonlinear. Hence, the determination of a global stabilizing controller is possible only when some stability assumptions are verified by the open-loop system $(u=0$, see [14, 24]). When these hypothesis are not ver- 
ified, it is only possible to achieve semi-global or local/regional stabilization. In this case, given a stabilizing matrix $K$, we associate a region of attraction to the equilibrium point $x_{e} \equiv 0$ of the system (1.5). The region of attraction corresponds to all initial conditions $x_{0} \in \mathbb{R}^{n}$ such that the corresponding trajectories of the system (1.5) converge asymptotically to the origin [12]. Since the determination of the exact region of attraction is practically impossible, a problem of interest is to ensure asymptotic stability for a set of admissible initial conditions $x_{0}$. Hence, from the considerations above, in this chapter, we are interested in studying the stabilization problem stated as follows.

P1. Maximize the bound on the maximal allowable sampling perioid $\mathscr{T}_{2}$, for which asymptotic stability of the closed-loop system (1.5) can be ensured for some set of admissible initial conditions.

P2. Given $\mathscr{T}_{1}, \mathscr{T}_{2}$, find $K$ and a set of admissible initial conditions, as large as possible, for which asymptotic stability of the closed-loop system (1.5) is ensured.

Regarding a networked control system, problem P2 can be seen as a "controller" design problem, which is defined from the network schedule constraints. On the other hand, problem P1 can be seen as a "network" design problem. In this case, the network designer will consider the maximum allowable jitter (for which stability of the system can be guaranteed) as a constraint in the network scheduling. Of course, when it is possible, the objective is the global stabilization of the closed-loop system. Otherwise, a set of admissible initial conditions, included in the region of attraction of the closed-loop system, has to be defined. This set can be seen as an estimate of the actual region of attraction and defines a region of "safe initialization" for the system.

In order to develop conditions to solve problems $\mathbf{P 1}$ and $\mathbf{P 2}$, in the sequel a particular notation is adopted. For all integers $k \in \mathbb{N}$, a function $\chi_{k} \in \mathbb{K}$ can be defined such that equation (1.5) can be equivalently represented by:

$$
\forall \tau \in\left[0, T_{k}\right],\left\{\begin{array}{l}
\chi_{k}(0)=x\left(t_{k}\right), \\
\chi_{k}(\tau)=x\left(t_{k}+\tau\right), \\
\dot{\chi}_{k}(\tau)=\frac{d}{d \tau} \chi_{k}(\tau)=A \chi_{k}(\tau)+\operatorname{Bsat}\left(K \chi_{k}(0)\right) .
\end{array}\right.
$$

In the sequel, we aim at designing constructive LMI conditions guaranteeing the stabilization of such systems.

\subsection{Preliminary lemmas and theorems}

\subsubsection{Modified sector condition}

Using the notation $\chi_{k}$ given in (1.6), the following dead-zone function is defined

$$
\psi\left(K \chi_{k}(0)\right)=K \chi_{k}(0)-\operatorname{sat}\left(K \chi_{k}(0)\right) .
$$


Note that, $\psi\left(K \chi_{k}(0)\right)$ corresponds to a decentralized dead-zone nonlinearity. Considering the function $\psi\left(K \chi_{k}(0)\right)$, the closed-loop system can be re-written as

$$
\dot{\chi}_{k}(\tau)=A \chi_{k}(\tau)+B K \chi_{k}(0)-B \psi\left(K \chi_{k}(0)\right), \quad \forall \tau \in\left[0, T_{k}\right] .
$$

Consider now a matrix $G \in \mathbb{R}^{m \times n}$ and define the polyhedral set

$$
\mathscr{S}=\left\{x \in \mathbb{R}^{n} ;\left|\left(K_{i}-G_{i}\right) x\right| \leq u_{0 i}, i=1, \ldots, m\right\} .
$$

The next Lemma from [9], concerning the nonlinearity $\psi\left(K \chi_{k}(0)\right)$ is recalled.

Lemma 1. Consider the function $\psi\left(K \chi_{k}(0)\right)$ defined in (1.7). If $\chi_{k}(0) \in \mathscr{S}$ then the relation

$$
\psi^{T}\left(K \chi_{k}(0)\right) U\left[\psi\left(K \chi_{k}(0)\right)-G \chi_{k}(0)\right] \leq 0,
$$

is verified for any matrix $U \in \mathbb{R}^{m \times m}$ diagonal and positive definite.

The result in Lemma 1 can be seen as a generalized sector condition. As will be seen in the sequel, differently from the classical sector condition (used for instance in [28]), this condition allows to obtain stability conditions directly in an LMI form. For notation simplicity, in the sequel we denote $\psi\left(K \chi_{k}(0)\right)$ as $\psi_{k}$.

\subsubsection{Asymptotic stability of saturated and sampled-data systems}

Let us first recall the following theorem taken from [22] on asymptotic stability of saturated sampled-data systems.

Theorem 1. Consider given matrices $K$ and $G$ in $\mathbb{R}^{m \times n}$ and any positive definite diagonal matrix $U$ in $\mathbb{R}^{m \times m}$. Let $T_{\min }$ and $T_{\max }, T_{\min }<T_{\max }$, be two positive scalars and $V: \mathbb{R}^{n} \rightarrow \mathbb{R}^{+}$be a function for which there exist real numbers $0<\mu_{1}<\mu_{2}$ such that

$$
\forall x \in \mathbb{R}^{n}, \quad \mu_{1}|x|^{2} \leq V(x) \leq \mu_{2}|x|^{2} .
$$

and such that, for all $i=1, \ldots, m$ and $x \in \mathbb{R}^{n}$

$$
x^{T}\left(K_{i}-G_{i}\right)^{T}\left(K_{i}-G_{i}\right) x \leq u_{0 i}^{2} .
$$

Then, the two following statements are equivalent:

(i) For all $k \in \mathbb{N}, T_{k} \in\left[\mathscr{T}_{1}, T_{\text {max }}\right]$, the increment of the Lyapunov function satisfies

$$
\Delta V(k)-2 T_{k} \psi_{k}^{T} U\left[\psi_{k}-G \chi_{k}(0)\right]<0 ;
$$

where $\Delta V(k)=V\left(\chi_{k}\left(T_{k}\right)\right)-V\left(\chi_{k}(0)\right)$.

(ii) There exists a continuous functional $\mathscr{V}_{0}:\left[0, T_{\max }\right] \times \mathbb{K} \rightarrow \mathbb{R}$ which satisfies for all $z \in \mathbb{K}$

$$
\forall T_{k} \in\left[T_{\min }, T_{\max }\right] \quad \mathscr{V}_{0}\left(T_{k}, z\right)=\mathscr{V}_{0}(0, z)
$$


and such that, for all $k \in \mathbb{N}, T_{k} \in\left[T_{\min }, T_{\max }\right]$ and $\tau \in\left[0 T_{k}[\right.$ and

$$
\dot{\mathscr{W}}\left(\tau, \chi_{k}\right)=\frac{d}{d \tau}\left[V\left(\chi_{k}(\tau)\right)+\mathscr{V}_{0}\left(\tau, \chi_{k}\right)\right]-2 \psi_{k}^{T} U\left[\psi_{k}-G \chi_{k}(0)\right]<0,
$$

Moreover, if one of these two statements is satisfied, then, for all initial conditions $x(0)=\chi_{0}(0)$ in the set $\mathscr{E}$ defined by

$$
\mathscr{E}=\left\{x \in \mathbb{R}^{n} ; \quad V(x) \leq 1\right\},
$$

the solutions to system (1.2) with the saturated and sampled control law (1.4) converge asymptotically to the origin.

Under the conditions of Theorem 1, it follows that the set $\mathscr{E}$ is included in the region of attraction of the closed-loop system (1.5). Note that this set is not necessarily positively invariant for the continuous-time system, but it is indeed for the discretetime system, i.e. if $\chi_{0}(0) \in \mathscr{E}$ it follows that $\chi_{k}(0) \in \mathscr{E}$, for all $k>0$. Nonetheless, the convergence of the continuous-time system trajectories to the origin is guaranteed for any initial condition belonging to $\mathscr{E}$, i.e. regional asymptotic stability is ensured.

\subsubsection{Integral inequality}

We recall here a result proved in [23], which provides a lower bound on a certain integral quadratic term.

Lemma 2. Let $\omega:[a, b] \rightarrow \mathbb{R}^{n}$ be a differentiable function over $(a, b)$ having square integrable first order derivative. Then, for all $R \in \mathbb{S}_{+}^{n}$, for all matrix $N \in \mathbb{R}^{3 n \times 2 n}$, the inequality

$$
\int_{a}^{b} \dot{\omega}^{T}(u) R \dot{\omega}(u) d u \geq \Omega^{T}\left[H e\{N M\}-(b-a) N\left[\begin{array}{cc}
R & 0 \\
0 & 3 R
\end{array}\right]^{-1} N^{T}\right] \Omega,
$$

holds where

$$
\Omega=\left[\omega^{T}(b) \omega^{T}(a) \frac{1}{b-a} \int_{a}^{b} \omega^{T}(u) d u\right]^{T}, \quad M=\left[\begin{array}{ccc}
I & -I & 0 \\
I & I & -2 I
\end{array}\right],
$$

Proof. This lemma refers to the Wirtinger-based integral inequality stated in [23]. Define the function $z$ and the associated quantity $\mathscr{I}(z)$ as follows

$$
z(u):=\dot{\omega}(u)-R^{-1}\left[I \quad\left(\frac{2 u-b-a}{b-a}\right) I\right] N^{T} \Omega \text { and } \mathscr{I}(z):=\int_{a}^{b} z^{T}(u) R z(u) d u .
$$

The proof consists in reinjecting the definition of $z$ in $\mathscr{I}(z)$ and in developing the integral. Some elementary integral calculus and an integration by parts show that 


$$
\mathscr{I}(z)=\int_{a}^{b} \dot{\omega}^{T}(u) R \dot{\omega}(u) d u-\Omega^{T}\left[\operatorname{He}\{N M\}-(b-a) N\left[\begin{array}{cc}
R & 0 \\
0 & 3 R
\end{array}\right]^{-1} N^{T}\right] \Omega,
$$

Finally, the positive definiteness of matrix $R$ ensures that $\mathscr{I}(z)$ is positive definite, which yields the result of Lemma 2.

Remark 1. The previous lemma presents another formulation of the Wirtinger-based integral inequality. Indeed choosing the particular matrix $N=\frac{1}{b-a} M^{T} R$ allows recovering the original Wirtinger-based integral inequality from [23].

The objective of this chapter is to design new stability and stabilization conditions expressed in terms of LMI based on Theorem 1 as proposed in [22] together with the use of the Wirtinger-based integral inequality proposed in Lemma 2.

\subsection{Stabilization of sampled-data systems under input saturation}

In this section, asymptotic stabilization conditions in a regional or local context of the sampled-data system (1.2) with the saturating control law given by (1.4) are derived from the results of Theorem 1 . These conditions allow the computation of a gain $K$ that ensures the asymptotic stability of the closed-loop system.

Theorem 2. For given positive scalars $T_{\min }<T_{\max }$ and $u_{0 j}, j=1, \ldots, m$, assume that there exist positive definite matrices $P, R \in \mathbb{S}^{n}$, a positive definite diagonal matrix $U \in \mathbb{S}^{m}$, two matrices $S_{1}, Q_{1} \in \mathbb{S}^{n}$, a symmetric matrix $X \in \mathbb{S}^{n+m}$, matrices $Y, S_{2}, Q_{2} \in \mathbb{R}^{n \times n}$ and $N \in \mathbb{R}^{(4 n+m) \times 2 n}$, two matrices $\tilde{K}$ and $\tilde{G} \in \mathbb{R}^{m \times n}$ and a positive scalar $\varepsilon$ that satisfy, for $\theta=T_{\min }, T_{\max }$

$$
\begin{aligned}
& \Psi_{1}(\theta)=\Pi_{1}(\theta)+\theta\left(\Pi_{2}+\Pi_{4}\right)<0, \\
& \Psi_{2}(\theta)=\left[\begin{array}{cc}
\Pi_{1}(\theta)+\theta\left(\Pi_{3}-\Pi_{4}\right) & \theta N \\
* & -\theta\left[\begin{array}{cc}
R & 0 \\
0 & 3 R
\end{array}\right]
\end{array}\right]<0, \\
& \Psi_{3 j}\left(u_{0 j}\right)=\left[\begin{array}{cc}
P\left(\tilde{K}_{j}-\tilde{G}_{j}\right)^{T} \\
* & u_{0 j}^{2}
\end{array}\right] \geq 0, \quad \forall j=1, \ldots, m,
\end{aligned}
$$

with

$$
\begin{aligned}
\Pi_{1}(\theta)= & \operatorname{He}\left\{M_{1}^{T} P M_{4}-M_{6}^{T} S_{2} M_{2}-N M_{8}+M_{5}^{T} \tilde{G} M_{2}\right\}-M_{6}^{T} S_{1} M_{6}-\theta M_{3} Q_{1} M_{3} \\
& +\operatorname{He}\left\{\left(\varepsilon M_{1}^{T}+M_{4}^{T}\right)\left(A Y M_{1}+B \tilde{K} M_{2}-Y M_{4}-B U M_{5}\right)\right\}-2 M_{5}^{T} U M_{5} \\
\Pi_{2}= & M_{4}^{T} R M_{4}+\operatorname{He}\left\{M_{4}^{T}\left(S_{1} M_{6}+S_{2} M_{2}\right)+M_{1}^{T}\left(Q_{1} M_{3}+Q_{2} M_{2}\right)\right\} \\
\Pi_{3}= & H e\left\{M_{3} Q_{2} M_{2}\right\}, \quad \Pi_{4}=M_{7}^{T} X M_{7},
\end{aligned}
$$

where $^{1}$

\footnotetext{
${ }^{1}$ These matrices are not of the same dimension.
} 


$$
\begin{aligned}
& M_{1}=\left[\begin{array}{lllll}
I & 0 & 0 & 0 & 0
\end{array}\right], M_{2}=\left[\begin{array}{lllll}
0 & I & 0 & 0 & 0
\end{array}\right], \quad M_{3}=\left[\begin{array}{lllll}
0 & 0 & I & 0 & 0
\end{array}\right],
\end{aligned}
$$

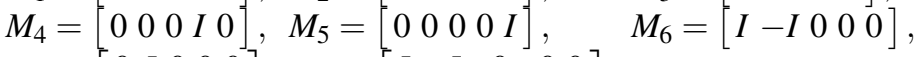

$$
\begin{aligned}
& M_{7}=\left[\begin{array}{lllll}
0 & I & 0 & 0 & 0 \\
0 & 0 & 0 & 0 & I
\end{array}\right], M_{8}=\left[\begin{array}{ccccc}
I & -I & 0 & 0 & 0 \\
I & I & -2 I & 0 & 0
\end{array}\right] \text {. }
\end{aligned}
$$

Then, for all initial conditions $x_{0}=x(0)$ belonging to the ellipsoidal set

$$
\mathscr{E}_{P}=\left\{x \in \mathbb{R}^{n} ; x^{T}\left(Y^{-T} P Y^{-1}\right) x \leq 1\right\},
$$

the corresponding trajectories of the system (1.2) under the saturated control law defined in (1.4) with $K=\tilde{K} Y^{-1}$ converge asymptotically to the origin for any asynchronous sampling satisfying (1.1).

Proof. Introduce a quadratic Lyapunov function candidate defined, for any $x$ in $\mathbb{R}^{n}$, by $V(x)=x^{T} \tilde{P} x$, where $\tilde{P}$ is a symmetric positive definite matrix from $\mathbb{S}^{n}$. Thus, the function $V$ satisfies (1.9) since it has a quadratic form.

Considering now the result of Theorem 1 , the idea is to prove that $\Delta V_{k}=$ $V\left(\chi_{k}\left(T_{k}\right)\right)-V\left(\chi_{k}(0)\right)-2 T_{k} \psi_{k}^{T} \tilde{U}\left[\psi_{k}-G \chi_{k}(0)\right]<0$, for all $k \in \mathbb{N}$. With this aim an appropriate functional $\mathscr{V}_{0}$ satisfying (1.11) and (1.12) must be chosen. A candidate of such a class of functionals is defined for all $\tau \in\left[0, T_{k}\right]$, as follows:

$$
\begin{aligned}
\mathscr{V}_{0}\left(\tau, \chi_{k}\right)= & \left(T_{k}-\tau\right)\left(\chi_{k}(\tau)-\chi_{k}(0)\right)^{T}\left[\tilde{S}_{1}\left(\chi_{k}(\tau)-\chi_{k}(0)\right)+2 \tilde{S}_{2} \chi_{k}(0)\right] \\
& +\left(T_{k}-\tau\right) \tau v_{k}^{T}(\tau)\left[\tilde{Q}_{1} v_{k}(\tau)+2 \tilde{Q}_{2} \chi_{k}(0)\right] \\
& +\left(T_{k}-\tau\right) \tau\left[\begin{array}{c}
\chi_{k}(0) \\
\psi_{k}
\end{array}\right]^{T} \tilde{X}\left[\begin{array}{c}
\chi_{k}(0) \\
\psi_{k}
\end{array}\right]+\left(T_{k}-\tau\right) \int_{0}^{\tau} \dot{\chi}_{k}^{T}(\theta) \tilde{R} \dot{\chi}_{k}(\theta) d \theta
\end{aligned}
$$

where $\tilde{S}_{1}, \tilde{Q}_{1}, \tilde{R}>0 \in \mathbb{S}^{n}, \tilde{S}_{2}, \tilde{Q}_{2} \in \mathbb{R}^{n \times n}$ and $\tilde{X} \in \mathbb{S}^{n+m}$ and where $v_{k}(\tau):=\frac{1}{\tau} \int_{0}^{\tau} \chi_{k}(s)$ $d s$.

The only difference with respect to the functional employed in [22] relies on the introduction of the function $v_{k}$. Introducing this function to the looped functional is necessary to fully take the advantages of the Wirtinger-based integral inequality provided in Lemma 2. It is clear that $v_{k}$ is continuous and differentiable over the interval $\left(0, T_{k}\right]$ and can be extended to the closed interval $\left[0, T_{k}\right]$, by noting that its limit, as $\tau$ goes to 0 , is $\chi_{k}(0)$. Moreover simple calculations show that

$$
\frac{d}{d \tau} v_{k}(\tau)=\frac{1}{\tau}\left(\chi_{k}(\tau)-v_{k}(\tau)\right)
$$

Obviously, this expression is only valid over the interval $\left(0, T_{k}\right]$ but can be extended by continuity to the interval $\left[0, T_{k}\right]$.

Since the functional $\mathscr{V}_{0}\left(\tau, \chi_{k}\right)$ is equal to zero at $\tau=0$ and $\tau=T_{k}$, the functional $\mathscr{V}_{0}$ satisfies looped condition (1.11). The end of the proof consists in showing that inequality (1.12) holds. Introduce the extended vector

$$
\tilde{\xi}_{k}(\tau)=\left[\chi_{k}^{T}(\tau) \chi_{k}^{T}(0) v_{k}^{T}(\tau) \dot{\chi}_{k}^{T}(\tau) \psi_{k}^{T}\right]^{T},
$$


for all $\tau \in\left[0, T_{k}[\right.$. Thanks to this augmented vector, an upper-bound of the derivative of $\mathscr{W}$ is given by

$$
\dot{\mathscr{W}}\left(\tau, \chi_{k}\right) \leq \tilde{\xi}_{k}^{T}(\tau)\left[\tilde{\Pi}_{1}\left(T_{k}\right)+\left(T_{k}-\tau\right) \tilde{\Pi}_{2}+\tau \tilde{\Pi}_{3}+\left(T_{k}-2 \tau\right) \tilde{\Pi}_{4}\right] \tilde{\xi}_{k}(\tau) .
$$

where

$$
\begin{aligned}
\tilde{\Pi}_{1}\left(T_{k}\right)= & \operatorname{He}\left\{M_{1}^{T} \tilde{P} M_{4}-M_{6}^{T} \tilde{S}_{2} M_{2}-\tilde{N} M_{8}\right\}-M_{6}^{T} \tilde{S}_{1} M_{6}-T_{k} M_{3} \tilde{Q}_{1} M_{3} \\
& +\operatorname{He}\left\{\left(M_{1}^{T} \tilde{Y}_{1}^{T}+M_{4}^{T} \tilde{Y}_{2}^{T}\right) M_{0}+M_{5}^{T} \tilde{U} G M_{2}\right\}-2 M_{5}^{T} \tilde{U} M_{5} \\
\tilde{\Pi}_{2}= & M_{4}^{T} \tilde{R} M_{4}+\operatorname{He}\left\{M_{4}^{T}\left(\tilde{S}_{1} M_{6}+\tilde{S}_{2} M_{2}\right)\right\}+\operatorname{He}\left\{M_{1}^{T}\left(\tilde{Q}_{1} M_{3}+\tilde{Q}_{2} M_{2}\right)\right\} \\
\tilde{\Pi}_{3}= & \operatorname{He}\left\{M_{3} \tilde{Q}_{2} M_{2}\right\}+\tilde{N}\left[\begin{array}{cc}
\tilde{R} & 0 \\
0 & 3 \tilde{R}
\end{array}\right]^{-1} \tilde{N}^{T}, \quad \tilde{\Pi}_{4}=M_{7}^{T} \tilde{X} M_{7}
\end{aligned}
$$

where $M_{0}=[A B K 0-I-B], \tilde{Y}_{1}, \tilde{Y}_{2} \in \mathbb{R}^{n \times n}, \tilde{N} \in \mathbb{R}^{4 n+m \times 2 n}$ and where the matrices $M_{i}$ are defined in Theorem 2. This inequality has been obtained by, first an application of Lemma 2, which ensures that, for all matrix $\tilde{N}$ in $\mathbb{R}^{4 n+m \times 2 n}$ to

$$
-\int_{0}^{\tau} \dot{\chi}_{k}^{T}(\theta) \tilde{R} \dot{\chi}_{k}(\theta) d \theta \leq \tilde{\xi}_{k}^{T}(\tau)\left[-\operatorname{He}\left\{\tilde{N} M_{6}\right\}+\tau \tilde{N}^{T}\left[\begin{array}{cc}
\tilde{R} & 0 \\
0 & 3 \tilde{R}
\end{array}\right]^{-1} \tilde{N}\right] \tilde{\xi}_{k}(\tau),
$$

and by noting that

$$
2\left(\chi_{k}^{T}(\tau) \tilde{Y}_{1}^{T}+\dot{\chi}_{k}^{T}(\tau) \tilde{Y}_{2}^{T}\right)\left(A \chi_{k}(\tau)-\dot{\chi}_{k}(\tau)+B K \chi_{k}(0)-B \psi_{k}\right)=0,
$$

for any square matrices $\tilde{Y}_{1}$ and $\tilde{Y}_{2} \in \mathbb{R}^{n \times n}$. This manipulation can be interpreted as the use of the descriptor approach introduced in [6] or the Finsler Lemma.

Since the matrices $K$ and $G$ are decision variables, the previous inequality is not an LMI. Indeed the matrix $\Pi_{1}$ contains products of matrices variables $\left(\tilde{Y}_{1}\right.$ with $K$ and $\tilde{Y}_{2}$ with $K$ ). There exists several transformations to derive an LMI condition that allows to compute a gain $K$, while guaranteeing that $\dot{\mathscr{W}}\left(\tau, \chi_{k}\right)<0$. In this chapter, a method inspired from [6] is applied. It relies on the fact that an underlying necessary condition of the previous condition to hold is the non singularity of the matrix $Y_{2}$. Additionally, $U$ is assumed to be a diagonal positive definite matrix. Thus it is possible to define the matrices $Y=\tilde{Y}_{2}^{-1}, U=\tilde{U}^{-1}$ and $\Xi=\operatorname{diag}\{Y, Y, Y, Y, U\}$. Consider the vector $\xi_{k}(\tau)=\Xi^{-1} \tilde{\xi}_{k}(\tau)$. Rewriting (1.18), using the new variable $\xi$ leads to

$$
\dot{\mathscr{W}}\left(\tau, \chi_{k}\right) \leq \xi_{k}^{T}(\tau) \Xi^{T}\left[\Pi_{1}+\left(T_{k}-\tau\right) \Pi_{2}+\tau \Pi_{3}+\left(T_{k}-2 \tau\right) \Pi_{3}\right] \Xi \xi_{k}(\tau) .
$$

From the definition of the matrices $M_{i}$ for $i=1, \ldots, 8$, one has

$$
\begin{aligned}
& M_{i} \Xi=Y M_{i}, i=1,2,3,4,6, M_{5} \Xi=U M_{5}, M_{7} \Xi=\left[\begin{array}{ll}
Y & 0 \\
0 & U
\end{array}\right] M_{7}, M_{8} \Xi=\left[\begin{array}{ll}
Y & 0 \\
0 & Y
\end{array}\right] M_{8}, \\
& M_{0} \Xi=A Y M_{1}+B K Y M_{2}-Y M_{4}-B U M_{5} .
\end{aligned}
$$


Setting now $Y_{1}=\varepsilon Y_{2}$ and considering the following change of variables $P=Y^{T} \tilde{P} Y$, $S_{i}=Y^{T} \tilde{S}_{i} Y, Q_{i}=Y^{T} \tilde{Q}_{i} Y$, for $i=1,2, X=\left[\begin{array}{ll}Y & 0 \\ 0 & U\end{array}\right]^{T} \tilde{X}\left[\begin{array}{ll}Y & 0 \\ 0 & U\end{array}\right], N=\Xi^{T} \tilde{N}\left[\begin{array}{ll}Y & 0 \\ 0 & Y\end{array}\right]$, $R=Y^{T} \tilde{R} Y, K=\tilde{K} Y$ and $G=\tilde{G} Y$, the following inequality is obtained:

$$
\dot{\mathscr{W}}\left(\tau, \chi_{k}\right) \leq \xi_{k}^{T}(\tau)\left[\Pi_{1}+\left(T_{k}-\tau\right) \Pi_{2}+\tau \bar{\Pi}_{3}+\left(T_{k}-2 \tau\right) \Pi_{4}\right] \xi_{k}(\tau) .
$$

where $\bar{\Pi}_{3}=\Pi_{3}+N^{T}\left[\begin{array}{cc}R & 0 \\ 0 & 3 R\end{array}\right]^{-1} N$ and $\Pi_{1}, \Pi_{2}, \Pi_{3}$ and $\Pi_{4}$ are defined in (1.17). To prove that $\dot{\mathscr{W}}$ is negative definite for all $\tau$, note that the right hand side of equation (1.21) is affine with respect to the variable $\tau$ in $\left[0, T_{k}\right]$. Then, by convexity, it suffices to ensure that the right hand side of (1.21) is negative for $\tau=0$ and $\tau=T_{k}$ (see [17] for more details). This fact leads to the inequalities

$$
\Pi_{1}+T_{k}\left(\Pi_{2}+\Pi_{3}\right)<0, \text { and } \Pi_{1}-T_{k} \Pi_{3}+T_{k} N R^{-1} N^{T}<0 .
$$

Applying the same argument on $T_{k}$ in the interval $\left[T_{\min }, T_{\max }\right]$ and by using the Schur's complement, conditions $\Psi_{1}(\theta)<0$ and $\Psi_{2}(\theta)<0$ given in (1.15) are obtained for $\theta=T_{\min }, T_{\max }$.

Right and left-multiplying (1.16) by $\operatorname{diag}\left\{Y_{2}, I\right\}$ and next applying Schur's complement, we conclude that (1.16) is equivalent to (1.10). Hence, it follows that $\mathscr{E}_{P} \subset \mathscr{S}$ and the condition (1.8) is verified for all $\chi_{k}(0) \in \mathscr{E}_{P}$.

Hence, by virtue of Theorem 1, conditions (1.15)-(1.16) ensures the asymptotic convergence of the trajectories to the origin, provided that $x(0) \in \mathscr{E}_{P}$.

Remark 2. The conditions from Theorem 2 are linear with respect to the matrices $A$ and $B$ which characterizes the dynamics of the system. Then a direct extension of this result can be provided in the case of systems with polytopic uncertainties defined by some positive scalars $\lambda_{i}$ 's such that $\sum_{i=1}^{M} \lambda_{i}=1$ and $[A B]=\sum_{i=1}^{M} \lambda_{i}\left[A_{i} B_{i}\right]$. Note that the $\lambda_{i}$ 's could either be constant parameters or time-varying but in both cases they are considered unknown.

\subsection{Optimization problems}

P1. Optimization of $T_{\max }$ for a given set of initial conditions: Thanks to Theorem 2, it is possible to include an optimization scheme in order to maximize the upper-bound of the sampling period for a given set of initial conditions. Define a region of admissible initial states as

$$
\mathscr{E}_{P_{0}}=\left\{x \in \mathbb{R}^{n}, \quad x^{T} P_{0} x \leq 1\right\} .
$$

Then, it is clear that the condition $\tilde{P}=Y^{-T} P Y^{-1}<P_{0}$ ensures that $\mathscr{E}_{P_{0}} \subset \mathscr{E}_{\tilde{P}}$. The following lemma is employed in the sequel. 
Lemma 3. Let $P$ be a positive definite matrix and $Y$ be a nonsingular matrix. Then the inequality $P_{0}>Y^{-T} P Y^{-1}$ holds if

$$
\left[\begin{array}{cc}
P_{0} & I \\
I & Y+Y^{T}-P
\end{array}\right]>0 .
$$

Proof. The proof can be found for example in [22].

Hence, given $T_{\min }$ and $u_{0}$, the idea is to find the maximal $T_{\max }$ for which it is possible to compute $K$ such that asymptotic stability is ensured for all initial conditions belonging to $\mathscr{E}_{P_{0}}$. An upper-bound on the maximal $T_{\max }$ can therefore be obtained from the following optimization problem

$$
\begin{gathered}
\max T_{\max } \\
\text { subject to } \\
(1.15),(1.16),(1.22) \text {. }
\end{gathered}
$$

Note that, from Lemma 3, the last inequality above ensures that $P<P_{0}$, which ensures that $\mathscr{E}_{P_{0}} \subset \mathscr{E}_{P}$.

P2. Maximization of the estimate of the region of attraction: For a given $u_{0}$ and an asynchronous sampling satisfying (1.1) with given $T_{\min }$ and $T_{\max }$, the objective is to determine $K$ that leads to the largest set of initial conditions for which the conditions of Theorem 2 are satisfied. In other words, we should find $K$ such that $\mathscr{E}_{P}$ is maximized considering some size criterion. For instance, we can maximize the minimal axis of $\mathscr{E}_{P}$, which corresponds to minimize the maximal eigenvalue of $P$. This can be accomplished from the following optimization problem

$$
\begin{gathered}
\min \delta \\
\text { subject to } \\
(1.15),(1.16), \\
{\left[\begin{array}{cc}
\delta I & I \\
I & \tilde{Y}+\tilde{Y}^{T}-P
\end{array}\right]>0 .}
\end{gathered}
$$

Note that, from Lemma 3 , the last inequality above ensures that $\tilde{P}<\delta I$, which ensures that $\lambda_{\text {max }}(\tilde{P})<\delta$.

Remark 3. For a fixed $\varepsilon$, the constraints in (1.23) are LMIs. Then the optimal solution of the previous problem can be easily approached by solving LMI-based problems on a grid in $\varepsilon$.

Remark 4. The result of Theorem 2 concerns the synthesis of a stabilizing gain $K$. Nonetheless, the conditions (1.15), and (1.16) can also be used for analysis purposes, when the gain $K$ is given. It suffices to replace $\tilde{K}$ by $K Y$. In this case, the problem (1.23) can be solved, to determine an estimate of the region of attraction of the closed-loop system or to determine a bound on the admissible sampling period jitter, respectively. Note that the conditions are still LMIs for a fixed $\varepsilon$. 


\subsection{Illustrative Examples}

\subsubsection{Example 1: Stability analysis for a given static output feedback controller}

We consider (1.2) with the following matrices, taken from [22] :

$$
\dot{x}(t)=\left[\begin{array}{cc}
0 & 1 \\
-2 & 0.1
\end{array}\right] x(t)+\left[\begin{array}{l}
0 \\
1
\end{array}\right] s a t_{u_{0}}\left(u\left(t_{k}\right)\right)
$$

and where $u_{0}=5$. The control gain $K$ is chosen as [1 0 . This system is unstable with a continuous-time state feedback control $u(t)=K x(t)$. However, it was proven in [20] that the closed-loop system using a sampled version of the same control law becomes stable if the sampling period becomes sufficiently large. We will show now that the same behavior appears even if the system is subject to input saturation.

In order to asses the stability of the closed-loop system with the given gain $K$, we can consider the two optimization problems (1.23) and (1.24) with the variable $\tilde{K}$ replaced by $K Y$ (see Remark 4).

Consider the first optimization problem P1. With $P_{0}=I$, the results of the optimization problem (1.23) with are summarized in Table 1.1. One can clearly see the considerable improvements with respect to [22] based on Jensen inequality.

\begin{tabular}{|c|c|c|c|c|c|}
\hline$T_{\min }$ & 0.25 & 0.4 & 0.8 & 1.2 & 1.5 \\
\hline$[5]$ & - & - & - & - & - \\
{$[22]$} & 0.37 & 0.71 & 0.97 & - & - \\
Theorem 2 & 0.44 & 1.01 & 1.33 & 1.48 & 1.54 \\
\hline
\end{tabular}

Table 1.1 Maximal allowable sampling period $T_{\max }$ for Example 1.

Consider now the second optimization problem P2. For $T_{\min }=0.8, T_{\max }=1$ and $u_{0}=5$. We aim at optimizing the size of the set of the allowable initial conditions. Solving problem (1.23) we obtained $\delta_{\text {min }}=1.2$ while in [22], the same minimization problem leads to $\delta_{\text {min }}=6.37$. Again, the improvements of the method proposed in the present chapter based on the Wirtinger-based integral with respect to the stability conditions from [22] based on Jensen inequality.

\subsubsection{Example 2: Stabilization using a static feedback controller}

We consider (1.2) with the following matrices (taken from [3], where $h=0$ ):

$$
\dot{x}(t)=\left[\begin{array}{ll}
1.1 & -0.6 \\
0.5 & -1.0
\end{array}\right] x(t)+\left[\begin{array}{l}
1 \\
1
\end{array}\right] s a t_{u_{0}}\left(u\left(t_{k}\right)\right),
$$


and where $u_{0}=5$ and $T_{\max }=1$. We aim here at optimizing the controller gain in order to maximize the set of allowable initial conditions. Applying the conditions from [22] with $\varepsilon=1$, it follows that the asymptotic stability of the system is ensured with the controller gain $K=[-1.74830 .5391]$ for any asynchronous sampling period characterized by $T_{\min }=0$ and $T_{\max }=1$. The obtained set of admissible initial conditions in this case is given by

$$
\mathscr{E}_{1}=\left\{x \in \mathbb{R}^{2} ; x^{T}\left[\begin{array}{rr}
0.4320 & -0.1332 \\
-0.1332 & 0.0411
\end{array}\right] x \leq 1\right\}, \quad \delta_{\min 1}=0.497 .
$$

Using now the conditions provided in the present chapter with $\varepsilon=1$, the optimal controller gain is $K=[-1.53550 .4735]$ and the set of acceptable initial conditions is

$$
\mathscr{E}_{2}=\left\{x \in \mathbb{R}^{2} ; x^{T}\left[\begin{array}{rr}
0.4319 & -0.1332 \\
-0.1332 & 0.0411
\end{array}\right] x \leq 1\right\}, \quad \delta_{\min 2}=0.497 .
$$

Since the results of the two lemmas are very close to each other, we can conclude that the use of the Wirtinger-based integral inequality does not help in improving this optimization problem for the stabilization.

\subsection{Conclusion}

This chapter proposes a novel constructive stabilization criterion for sampled and saturated controlled systems based on the discrete-time Lyapunov theorem. The difference with respect to the previous research remains in the use of the Wirtingerbased integral inequality in order to reduce the conservatism of the conditions. Moreover, as illustrated in the example the method provides less conservative results than existing approaches reported in the literature at least for the analysis.

\section{References}

1. Alamo T, Cepeda A, Limon D, and Camacho E F (2006) Estimation of the domain of attraction for saturated discrete-time systems. International Journal of Systems Science, 37(8):575583.

2. Åstrom KJ, and Wittenmark B (1984) Computer Controlled Systems. Prentice-Hall.

3. Cao Y, Lin Z, and Hu, T (2002) Stability analysis of linear time-delay systems subject to input saturation, IEEE Trans. on Circuit and Systems I, 49:233-240.

4. Fridman, E (2010) A refined input delay approach to sampled-data control. Automatica, 46(2):421-427.

5. Fridman E, Seuret A, and Richard J-P (2004) Robust sampled-data stabilization of linear systems: An input delay approach. Automatica, 40(8):1141-1446.

6. Fridman E, and Shaked U. (2002) A descriptor system approach to $H^{\infty}$ control of linear timedelay systems. IEEE Trans. on Automatic Control, 47(2):253-270.

7. Gomes da Silva Jr. J M, Seuret A, Fridman E, and Richard J-P (2011) Stabilisation of neutral systems with saturating control inputs. International Journal of Systems Science, 42(7):10931103. 
8. Gomes da Silva Jr. JM, and Tarbouriech S. (2001) Local stabilization of discrete-time linear systems with saturating controls: an lmi-based approach. IEEE Trans. on Automatic Control, 46(1):119-124.

9. Gomes da Silva Jr. JM, and Tarbouriech S. (2005) Anti-windup design with guaranteed regions of stability: an LMI-based approach. IEEE Trans. on Automatic Control, 50(1):106111.

10. Hespanha JP, Naghshtabrizi P, and Xu Y (2007) A survey of recent results in networked control systems. Proceedings of the IEEE, 95(1):138-162.

11. Hindi H, and Boyd S (1998) Analysis of linear systems with saturation using convex optimization. In Proc. of the IEEE Conference on Decision and Control, pages 903-908, Tampa or, USA.

12. Khalil H K (1996) Nonlinear Systems. Prentice Hall, 1996.

13. Kiyama T, and Iwasaki T (2000) On the use of multi-loop circle for saturating control synthesis. Systems \& Control Letters, 41:105-114.

14. Lin Z, and Saberi A (1993) Semi-global exponential stabilization of linear systems subject to input saturation via linear feedback. System \& Control Letters, 21:225-239.

15. Mazenc, F, Mondié, S, and Niculescu, S-I (2003) Global Asymptotic Stabilization for Chains of Integrators with a Delay in the Input. IEEE Transactions on Automatic Control, Vol. 48(1):57-63.

16. Mazenc, F, Mondié, S, and Niculescu, S-I (2004) Global Stabilization of Oscillators with Boun- ded Delayed Input. Systems \& Control Letters, 53(5):415-422.

17. Naghshtabrizi P, Hespanha JP, and Teel AR (2008) Exponential stability of impulsive systems with application to uncertain sampled-data systems. Systems \& Control Letters, 57(5):378385.

18. Niculescu S-I, Dion J-M and Dugard L. (1996) Robust stabilization for uncertain time-delay systems containing saturating actuators. IEEE Trans. on Automatic Control, 41(5):742-747.

19. Oucheriah S (1996) Global stabilization of a class of linear continuous time delay systems with saturating controls. IEEE Trans. on Circuit and Systems I, 43(12):1012-1015.

20. Seuret A (2012) A novel stability analysis of linear systems under asynchronous samplings. Automatica, (1):177 182.

21. Seuret A, and Briat C (2014) Stability analysis of uncertain sampled-data systems with incremental delay using looped-functionals, submitted to Automatica.

22. Seuret A, and Gomes da Silva Jr. JM (2012) Taking into account period variations and actuator saturation in sampled-data systems, Systems \& Control Letters, 61(12), 1286-1293.

23. Seuret A, and Gouaisbaut F (2013) Wirtinger-based integral inequality: Application to timedelay systems. Automatica, 49(9):2860-2866.

24. Sussmann HJ, Sontag ED, and Yang Y (1994) A general result on the stabilization of linear systems using bounded controls. IEEE Trans. on Automatic Control, 39(12):2411-2425.

25. Suh YS (2008) Stability and stabilization of nonuniform sampling systems. Automatica, 44(12):3222-3226.

26. Tarbouriech S, Garcia G, Gomes da Silva Jr. JM, and Queinnec I (2011) Stability and Stabilization of Linear Systems with Saturating Actuators. Springer, 2011.

27. Tarbouriech S, and Gomes da Silva Jr. J M (2000) Synthesis of controllers for continuoustime delay systems with saturating controls via LMIs. IEEE Trans. on Automatic Control, 45(1):105-111.

28. Tarbouriech S, Gomes da Silva Jr. JM, and Garcia G (2003) Delay-dependent anti-windup loops for enlarging the stability region of time-delay systems with saturating inputs. Trans. ASME - J. of Dyn. Syst., Meas.and Contr., 125(1):265-267.

29. Yamamoto Y (1994) A function space approach to sampled data control systems and tracking problems. IEEE Trans. on Automatic Control, 39(4):703-713.

30. Zhang L, Boukas E K and Haidar A (2008) Delay-range-dependent control synthesis for time-delay systems with actuator saturation. Automatica, 44(10):2691-2695.

31. Zhou B., Zheng WY, and Duan G-R (2011) An improved treatment of saturation nonlinearity with its application to control of systems subject to nested saturation. Automatica, 47:306315 . 